\title{
Analysis and Performance Evaluation of Software System Usability
}

\author{
Piyush Jain \\ Amity University \\ Noida, India
}

\author{
Sanjay Kumar Dubey \\ Amity University \\ Noida , India
}

\author{
Ajay Rana ,PhD \\ Amity University \\ Noida , India
}

\begin{abstract}
Usability is the methodology used to determine the software performance. Usability is basically used to determine the strength and weakness of an application from the end user point of view. Questionnaires have been used to evaluate user interface of different application. This paper is a survey and analysis of the performance of Microsoft office 2003-2007 packages. We have considered this product because of its large scale usage in professional and academics. In this paper we have presented the survey conducted over different professional and also analysis of those result.
\end{abstract}

\section{General Terms}

Software Usability, SUMI

\section{Keywords}

\section{Usability, Performance, System, Model}

\section{INTRODUCTION}

The demand of quality models is increasing day by day. Usability is one of the important factor in each quality model. There are different perceptive to evaluate the usability. In the ISO/IEC 9126-4 (2001) standard, the difference between usability and the quality in use is a matter of context of use. Specifically, when usability is evaluated, the focus is on improving the user interface while the context of use is treated as a given. This means that the level of usability achieved will depend on the specific circumstances in which the product is used. In contrast, when quality in use is evaluated, any component of context of use may be subject to change or modification. This is why Bevan and Macleod viewed usability as a result of use of a computer tool in a particular context [10]. Specifically, they assume that quality in use can be measured as the outcome of interactions with a computer system, including whether intended goals of the system are achieved (effectiveness) with the appropriate expenditure of resources (e.g., time, mental effort) in way the user finds acceptable (satisfaction).

Usability is defined as an ease with which a human can understand and learn the manmade object. The object may be a hardware or software. If it is a software then we term usability as software usability. Usability plays an important role in every phase of designing. Usability cannot be measured directly but is quantified by using some quantified measures such as number of error reported. Usability engineering is an research methodology which is use to design a quality product.

According to A. Seffah et al [12] standard also distinguished external versus internal quality and defined related metrics. Internal quality generally concerns properties of the non executable portion of a software product during its development, and metrics for internal quality generally concern the quality of intermediate deliverables, such as the source code for a prototype version. In contrast, external quality concerns the behavior of the computer system of which the software product is a part. Metrics for external quality can be obtained only by executing the software product in the system environment for which the product is intended. Metrics in ISO/IEC 9126-4 (2001) are also classified in terms of level of measurement (i.e., nominal, ordinal, interval, or ratio) and measurement type (e.g., count variables [the number of times an event occurs], elapsed time, size [source size, function size, etc.]).[12]

ISO computer science society has written (separately) about a framework of system acceptability, where usability is a part of "usefulness" and which has the following factor [2] .

Table 1: Usability and its factors

\begin{tabular}{|l|l|l|}
\hline SNO & FACTORS & DEFINATION \\
\hline 1 & LEARN-ABILITY & $\begin{array}{l}\text { Attributes that describe the } \\
\text { relative ease for the users to } \\
\text { learn the application. }\end{array}$ \\
\hline 3 & MEMORABILITY & $\begin{array}{l}\text { This attribute give an idea } \\
\text { whether the system is } \\
\text { working according to the } \\
\text { requirement or not. } \\
\text { design after a period of } \\
\text { not using it, how easily } \\
\text { can they re-established }\end{array}$ \\
\hline 4 & ERRORS & $\begin{array}{l}\text { Whether the system is } \\
\text { generating error or not. If } \\
\text { yes how intense the error } \\
\text { are. }\end{array}$ \\
\hline 5 & SATISFACTION & $\begin{array}{l}\text { The attribute tell that } \\
\text { whether the design is } \\
\text { pleasant }\end{array}$ \\
\hline
\end{tabular}

Usability is a technique of determining an effective technique of judging the popularity of software among the customer. Usability is the measure of performance of software which includes the interaction oriented, user oriented, product oriented and formal view of software requirement.

In view of the above context, this paper focuses on the analysis, evaluating and comparison of usability of any software system. Microsoft office products are being extensively used daily by many professional. Microsoft office 
excel is extensively used as a spreadsheet for various purpose. The reason why MS excel is been used are [3] :-

i. MS excel spreadsheet is compatible with other excel users too.

ii. MS excel is compatible with other financial and data manipulation software's.

iii. Always an online help system is provided and it is robust in nature too.

iv. Ease to use which one of the important factor considered while designing a software.

We generally use two version of Microsoft office ie Excel 2003 and Excel 2007. The Microsoft office suit has been recently released the $12^{\text {th }}$ version of its office package "MS office 2007" [3]. Most of the customers are using MS office 2003 package, but after the release of MS office 2007 most of the customer has started working in 2007 version. The reason for that is more improved functionality and additional features. But in presence of striking feature there are some limitations that exist in Microsoft Office 2007. Some of the limitations are listed as below [3] :-

- After installing Microsoft 2007 excel gets aborted directly with a message that the program cannot be used if the terminal services are enabled.

- The menu items do not display well using Microsoft Magnifier.

- In the Options dialog box (Tools menu), using the $\mathrm{TAB}$ key to navigate to certain buttons changes the focus to the entire dialog box

This paper presents performance evaluation of MS excel by comparing MS excel 2003 and 2007. We are mainly concerned in evaluating those features that are essentials and those which are not. The usability study evaluates the pros and cons regarding a software so that the software is properly rated among others.

This paper has conducted a study on usability of MS excel which is divided into two survey phases. The first phase of survey we have introduced the research concepts. In the second phase of the research we will comment of the usability of MS Excel 2003 and 2007. We will use the SUMI evaluation technique for determining the usability of the MS office package.

\section{LITERATURE SURVEY}

The usability is a survey based study where multiple factors and tools are considered to calculate the usability. Usability is calculated on the basis of multiple internal and external metrics as explained by all software quality model.

The author Abdul Rauf et al from National University of Computer \& Emerging Science, Islamabad has already mentioned much more about usability survey. In his work he has conducted an survey on Microsoft Office Word. The author has evaluated the usability study of Microsoft Office 2003 and 2007 where he has analyzed and evaluated its performance [1].
The author Ahmed Seffah Mohammad et al in this paper has designed a integrated tool for measuring usability which is based on the factors affecting the software quality. The author has designed 10 factors for evaluating usability which are further dived into 127 sub-factors [12]

The author Drs. Erik P.W.M. has evaluated usability on the basis of SUMI (software usability measurement inventory) Questionnaire. The author has applied the SUMI over multiple software's to determine the reliability of the SUMI questionnaire. Through this work the author has reveal that the SUMI scores at least $80 \%$ reliable [14].

The author Jagat Sesh Challa et al in this paper has work with the different factors affecting the quality. Based on that the author has designed an integrated model based on fuzzy metric. In that model the author has evaluated the usability based on some external metric. The metric are quantified by using membership function. The result showed that the model is better in evaluating software quality better than other models [15].

The author K. Christoph Homberg in his work presented that the usability questionnaires called Iso Metrics which is based on the international standard ISO 9241[4]. The survey was conducted over an Hospital Information System. Iso metric questionnaire help to evaluate the essentials features of the information system. The author has used multiple question regarding the performance and is able to determine specific point of view regarding this information system.

The author Mells et al introduced a traditional office application which include Visual Studio tools for Office (VSTD) technology. This tool has significant advantage for the environmental management user sector [5].

The author Miles Macleod has done usability context analysis (UCA) where a practical co-operative method is applied for identifying and recording contextual aspects of usability in system development. UCA has provided a method to work most effectively as a co-operative process for bring together a range of people who have stake in the development or use of an IT product or interactive system [13].

The author Nigel Bevan has designed an usability measuring tool ESPRIT MUSIC [9] (Measurement of usability in context). This tool can be used to predict, ensure and improve the product quality. This can also be used to decide whether the software should be accepted or not [10][8].

The author Sanjay Kumar Dubey has discussed some of the important issues for calculating usability. The author has also described some of the points that must be considered while considering any usability method. The paper has also discussed the entire model been designed till now[16].

All the above references either explain the tool for measuring usability or explaining some metrics for evaluating usability.

\section{RESEARCH SURVEY}

This survey deals with the usability study of Microsoft office excel 2003 and 2007. Usability evaluation of any software will help the developer to recognize the limitation faced by 
user of specific software [7] . After the evaluation of limitation of the software the developer will tries to solve the problem. Till now the usability evaluation technique includes these different approaches depending upon user's experience. They are used based evaluation, expert evaluation and hybrid approaches [6].

The survey conducted in this paper is divided in two phases. Each phases has specific significance. The flow of the survey can be defined by the given flow diagram in figure1. The figure will reflect the different phases of the survey and how the comparison is done.

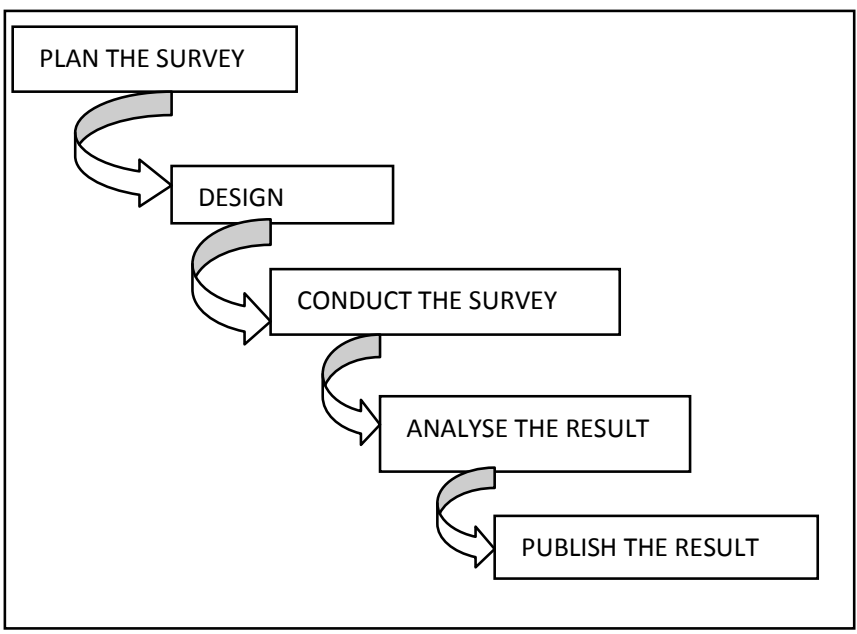

Fig 1: A life cycle representing the survey phases.

\subsection{Survey Phase 01}

During the first phase of survey we have tried to analyze and compare the latest version of MS office excel 2003 with MS office 2007. This survey has given us many relevant data regarding the essential feature of both the product. Before conducting the survey we have to first categorize the end user of the product because they are the person who has to give judgment on the product. We have three categories of users. The categories are:-

- $\quad$ The user belonging to computer related field.
- $\quad$ The user having working knowledge in MS office excel.

- The user belonging to non computer field.

We have surveyed sixty persons from different university/office/organization and are asked to fill the questionnaire. The result is evaluated and among them twenty experts are selected who have more knowledge of the MS Excel package and are asked to answer the given questionnaire. The questionnaire consisted of thirty questions which reflect the functional features of MS office excel 2003 and 2007.

\subsection{Survey Phase 02}

In the second phase of the survey we have chosen expert from the sixty persons and are asked to answer the questionnaire of the second phase. We asked them to fill the SUMI [17] questionnaires which are used to evaluate the user satisfaction level [8]. In this survey we have evaluated an interesting fact that MS Excel 2007 provides more flexibility then MS excel 2003. We have considered the important features of the MS Excel packages and asked them to evaluate its usability.

According to the paper written by Nigel Beven [10] Neilson has said that "about $85 \%$ of the usability problems should be discovered". Finally we can say that the survey we have conducted is enough to judge the performance of the software.

\section{Survey Analysis}

This survey is capable of revealing the fact that MS office Excel is an useful and most efficient software for general purpose work. People are satisfied with the MS office packages and prefer it most as compared to other similar Software packages. The survey also reveal that MS Office Excel 2007 is no doubt providing more features and functionality as compared to MS Office Excel 2003 is more convenient to use and provide more satisfaction to the user. The survey reflects two striking factors required to judge any software. They are

- Personal satisfaction

- Personal efficiency

MS Office Excel 2007 is capable to fulfill the professional efficiency and is able to meet the personal satisfaction also.

Table 2: Result Survey Phase 02

\begin{tabular}{|c|l|c|c|}
\hline SNO & \multicolumn{1}{|c|}{ FEATURES } & MS OFFICE EXCEL 2007 & MS OFFICE EXCEL 2003 \\
\hline $\mathbf{1}$ & Insert Row \& Column & 9 & 8 \\
\hline $\mathbf{2}$ & Row Height Adjustment & 10 & 8 \\
\hline $\mathbf{3}$ & Formula & 8 & 6 \\
\hline $\mathbf{4}$ & Header \& Footer & 8 & 9 \\
\hline $\mathbf{5}$ & Intermediate Formatting of Text & 8 & 6 \\
\hline
\end{tabular}




\begin{tabular}{|l|l|c|c|}
\hline $\mathbf{6}$ & Hyperlink & 9 & 8 \\
\hline $\mathbf{7}$ & Graphics \& Chart & 8 & 6 \\
\hline $\mathbf{8}$ & Margin \& Page Setup & 9 & 9 \\
\hline $\mathbf{9}$ & Page Break \& Section Break & 8 & 6 \\
\hline $\mathbf{1 0}$ & Macros & 8 & 1 \\
\hline
\end{tabular}

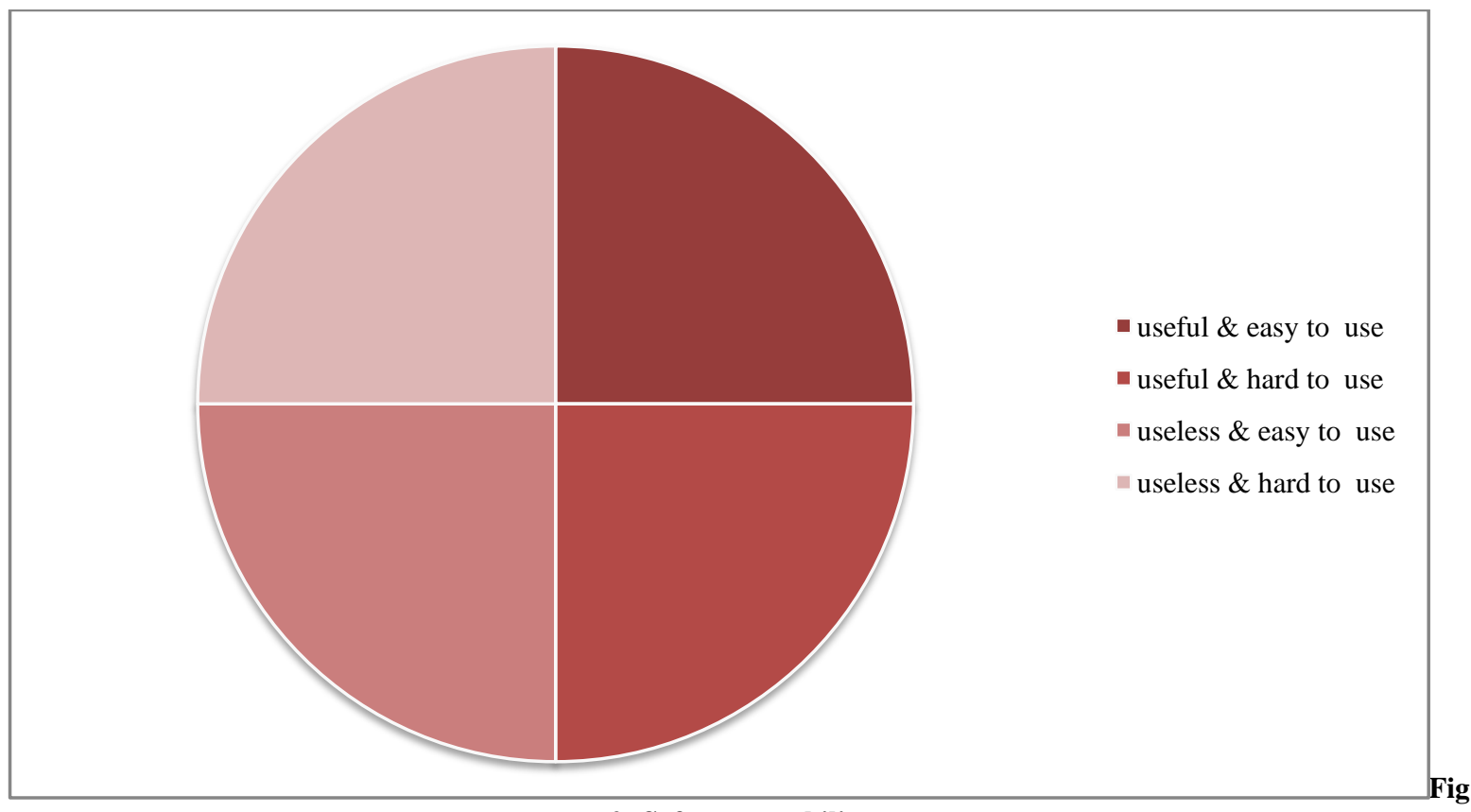

2: Software usability

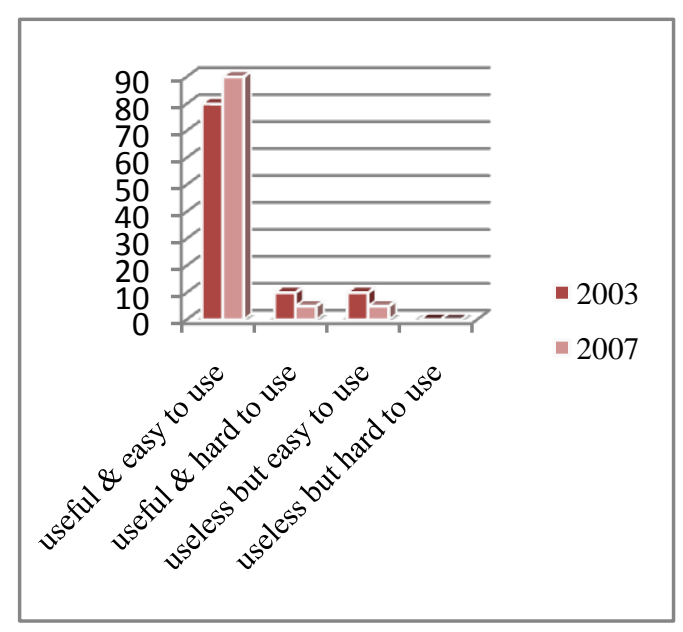

Fig 3: Insert Row And Column

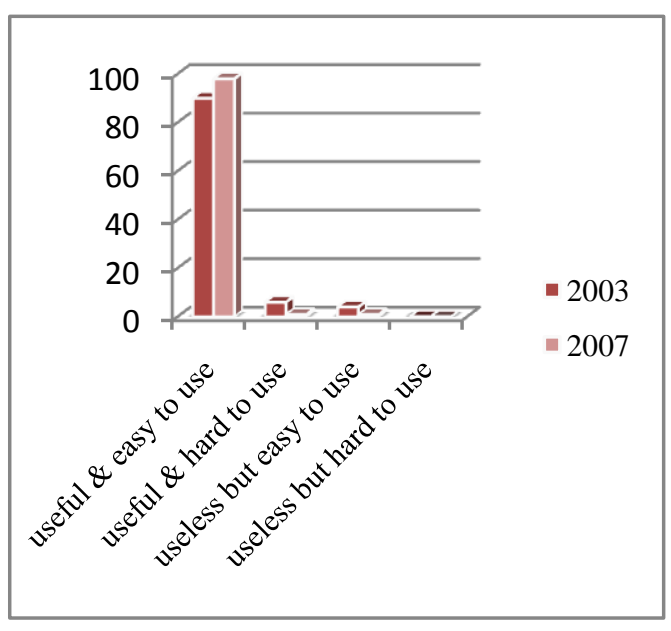

Fig 4: Row Height Adjustment 


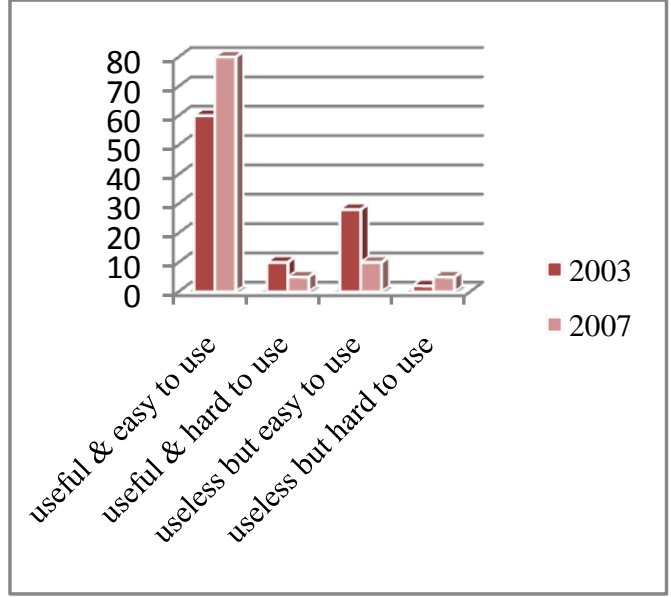

Fig 5: Formula

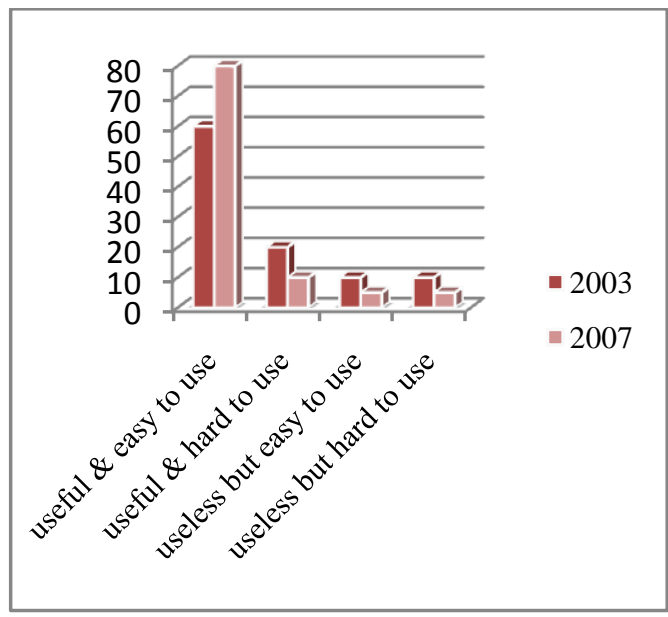

Fig 7: Intermediate Text Formatting

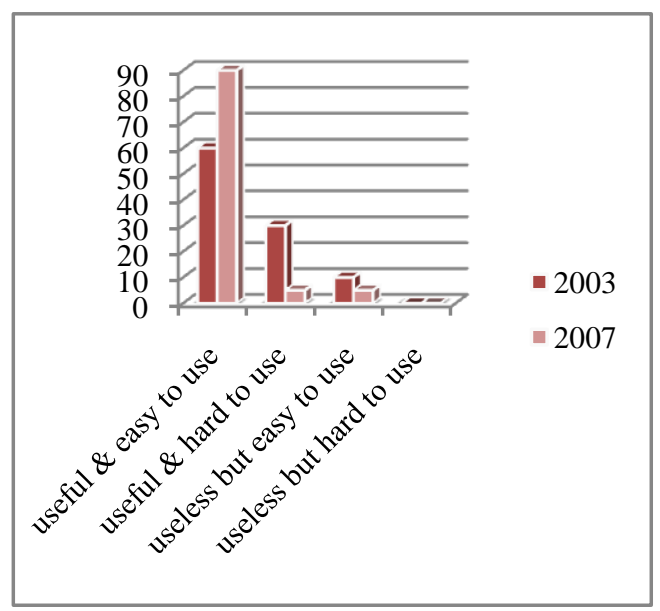

Fig 9: Graphics and Chart

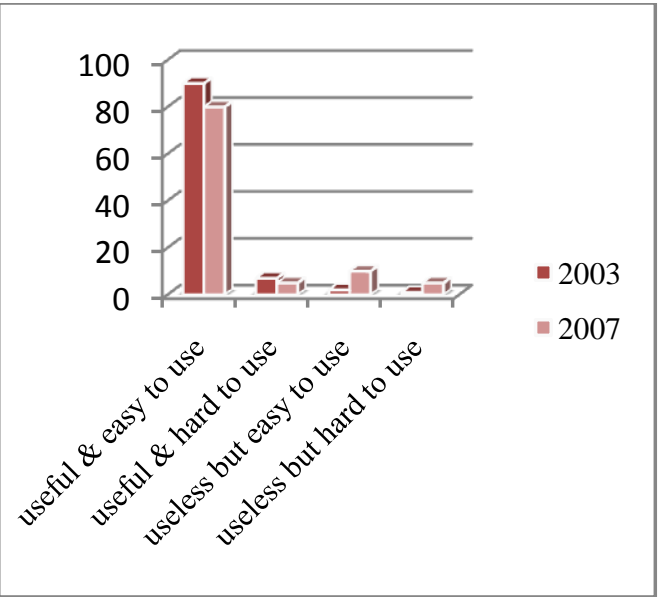

Fig 6: Page Header and Footer

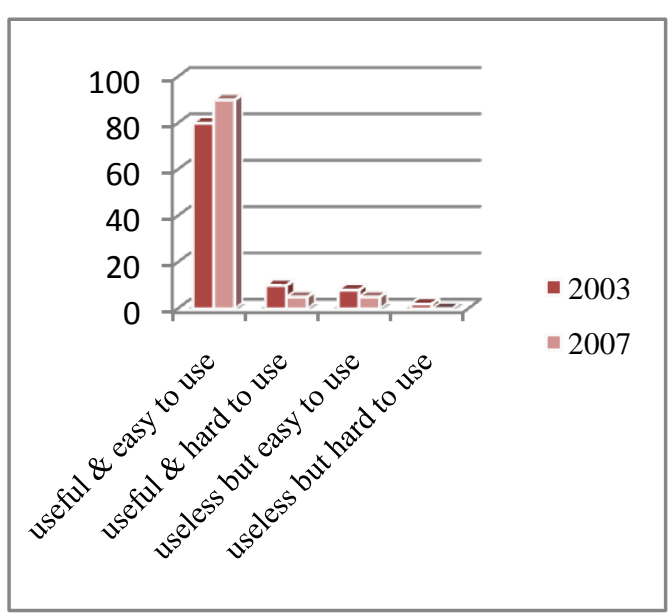

Fig 8: Hyperlink

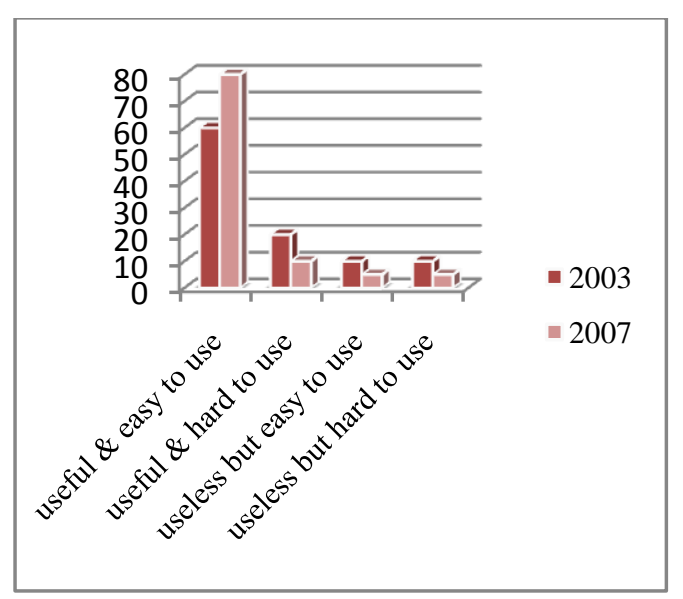

Fig 10: Margin \& page setup 


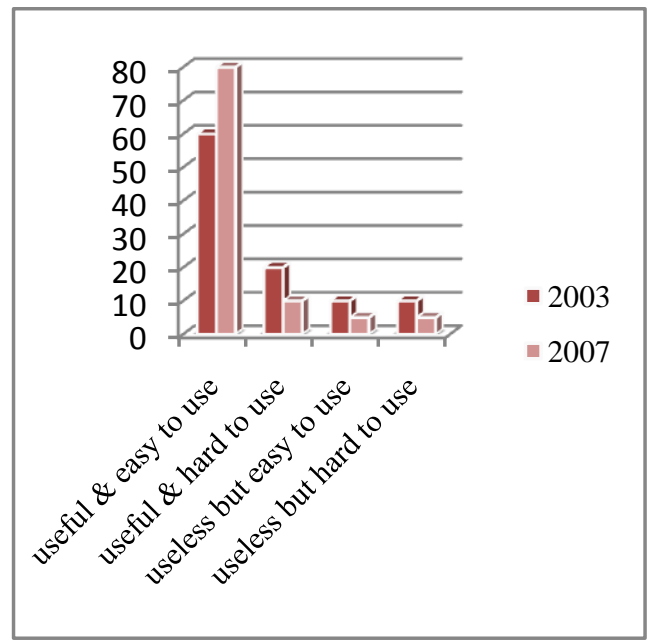

Fig 11: Page \& Section Break

\section{RESULT AND DISCUSSION}

Usability is the measure of software performance and user satisfaction. This survey help to reveal the fact that MS Excel is the more prefer software package for daily work. The survey also reveals that the user always think of their personal satisfaction. By this survey we reflect the fact that MS office excel 2007 is better in performance then excel 2003. This provide more functional and efficiency as compared to MS office 2003.

\subsection{Limitation of Study}

Usability is used to reveal the performances of MS office excel but still this survey has some limitations also. This limitation is listed as below:-

Although we have conducted the survey efficiently, but it is done with the help of non technical users. So the usability result revealed is not guaranteed to be $100 \%$ correct.

A lots of contradiction arises while comparing both MS Office Excel 2003 and 2007.

\section{REFERENCES}

[1] Survey based usability evaluation of MS WORD Abdul Rauf, Sadia Batool, Shafiq Ur Rehman, Arshad Ali Shahid

[2] Introduction to usability , Jacksob Nielson 2010-06-01

[3] http://www.microsoftoffice.com.

[4] Hamborg KC, Vehse B, Bludau HB Questionnaire based usability evaluation of hospital information systems 2004

[5] Steven Furnell, Dimitris Katsabas, Paul Dowland, Fraser Reid: A Practical Usability Evaluation of Security Features in End-User

[6] Ijo, R and Mannter, J Are non expert usability evaluation valuable $18^{\text {th }}$ international symposium MFT on human factor in Telecommunication.

[7] Freiberg M Baurneister J A survey on usability evaluation technique and an analysis of their actual application.

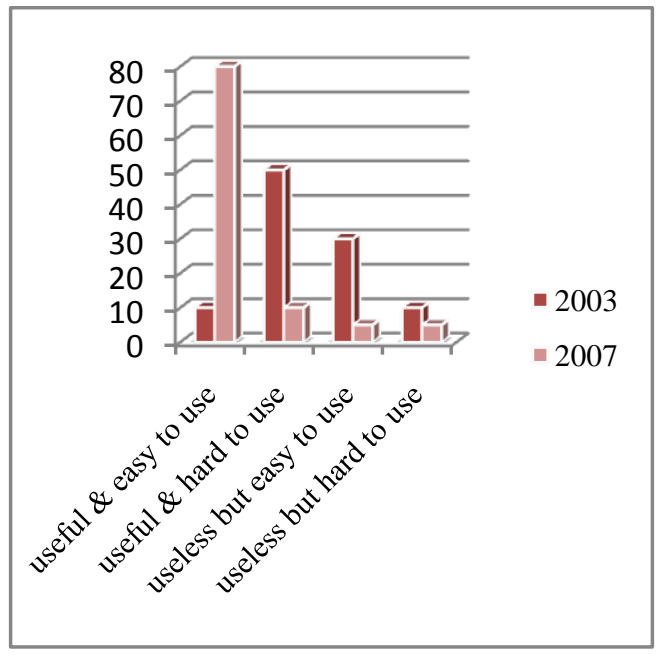

Fig 12: Macros

[8] Bevan, N. 1995. Measuring usability as quality of use, Software Quality Journal 4: 115-130.

[9] Bevan, N. and Azuma, M. 1997. Quality in Use: Incorporating human factors into the software engineering.

[10] Bevan, N. and Macleod, M. 1994. Usability measurement in context, Behavior and Information Technology

[11] Bevan, N. and Schoeffel, R. 2001. A proposed standard for consumer product usability, Proceedings of $1^{\text {st }}$

[12] Usability measurement and metrics: A consolidated model Ahmed Seffah Mohammad Donyaee - Rex B. Kline $\cdot$ Harkirat K. Padda Software Qual J (2006)

[13] Usability in Context: Improving Quality of Use Miles Macleod National Physical Laboratory, DITC, Teddington, Middlesex, TW11 0LW, UK Proceedings of the International Ergonomics Association 4th International Symposium on Human Factors inOrganizational Design and Management, Stockholm, May 29 - June 1 1994). Amsterdam, Elsevier / North Holland.

[14] Low Cost Usability Testing Drs. Erik P.W.M. van Veenendaal CISA Improve Quality Services BV / Eindhoven University of TechnologyPublished in: Software Quality and Software in Internet Time, Springer Publishing Improve Quality Services BV, Waalreseweg 17, 5554 HA Valkenswaard.

[15] Integrated Software Quality Evaluation: A Fuzzy MultiCriteria Approach Jagat Sesh Challa, Arindam Paul, Yogesh Dada, Venkatesh Nerella, Praveen Ranjan Srivastava and Ajit Pratap Singh Journal of Information Processing Systems, Vol.7, No.3, September 2011.

[16] Issues in Usability Measurement for Object-Oriented System Sanjay Kumar Dubey and A. K. Malviya, Amity University NOIDA, (U.P.) 